\title{
Prognostic Factors and Therapeutic Strategies of Thyroid Carcinomas Originating from Follicular Cells
}

\author{
${ }^{1}$ Yasuhiro Ito, ${ }^{2}$ Akira Miyauchi \\ ${ }^{1}$ Staff Surgeon, Department of Surgery, Kuma Hospital, Shimoyamate-dori, Chuo-ku, Kobe, Japan \\ ${ }^{2}$ Director, Department of Surgery, Kuma Hospital, Shimoyamate-dori, Chuo-ku, Kobe, Japan
}

Correspondence: Yasuhiro Ito, Staff Surgeon, Staff Surgeon, Department of Surgery, Kuma Hospital, 8-2-35, Shimoyamatedori, Chuo-ku Kobe, 650-0011 Japan, Phone: 81-78-371-3721, Fax: 81-78-371-3645, e-mail: ito01@kuma-h.or.jp

\begin{abstract}
There are three types of thyroid carcinoma originating from follicular cells, papillary carcinoma (PTC), follicular carcinoma (FTC) and anaplastic carcinoma (ATC). PTC and FTC have generally indolent nature but ATC, which is believed to arise from PTC and FTC, are very progressive and display a dire prognosis. PTC and FTC are called differentiated carcinoma and regarded as a single group, but biological characteristics of these two types of carcinoma significantly differ. PTC frequently metastasizes to the regional lymph nodes and FTC generally metastasizes to distant organs such as the lung and bone. Most PTC can be diagnosed on preoperative imaging studies and fine needle aspiration biopsy (FNAB) and can be treated as malignancy. However, it is difficult to diagnose FTC preoperatively and most FTC are diagnosed on postoperative pathological examination. In this review, we describe prognostic factors of PTC and FTC and their therapeutic strategies. Furthermore, recent advances of treatment for ATC are also described.
\end{abstract}

Keywords: Thyroid carcinoma, prognostic factor, papillary, follicular, anaplastic.

\section{INTRODUCTION}

Thyroid carcinoma arising from follicular cells consists of three histological types, papillary carcinoma (PTC), follicular carcinoma (FTC), and anaplastic carcinoma (ATC). PTC and FTC are often called differentiated carcinoma (DTC) collectively, but their biological characteristics are quite different. PTC frequently metastasizes to the lymph node but FTC metastasizes predominantly to distant organs. Generally, these carcinomas have indolent natures but cases having certain characteristics show dire prognosis. Studies for prognostic factors of PTC and FTC are very important to accurately discriminate highrisk cases from others, but to date, most studies on this issue have investigated for PTC and FTC in a single group as DTC. On the bases of the difference in biological characteristics of these carcinomas, it is preferable to identify prognostic factors for disease-free survival (DFS) and cause specific survival (CSS) of patients with PTC and FTC separately. ATC is believed to arise from DTC with a very aggressive behavior, which should be considered high risk regardless of clinicopathological characteristics. However, evaluation of biological features at the time of diagnosis is still important to decide therapeutic strategy for patients and predict whether patients can expect long-term survival. In this review, prognostic factors of thyroid carcinomas of three types are described separately on the basis of recent publications, including ours, and their therapeutic strategies are discussed.

\section{PTC}

PTC can generally be diagnosed by cytological study, which means that most cases are diagnosed as PTC before surgery. The recent prevalence of ultrasonography and ultrasonography-guided fine needle aspiration biopsy (FNAB) enabled us to detect and diagnose PTC in an early phase. Ultrasonography on mass screening for thyroid and carotid artery can detect small thyroid nodules measuring $3 \mathrm{~mm}$ or larger and many of them are diagnosed as PTC on FNAB, indicating that the incidence of surgery for PTC of small size is increasing. Furthermore, small lymph node metastases that are not palpable can be diagnosed on ultrasonography on the basis of typical appearance ${ }^{1}$ and on FNAB followed by thyroglobulin measurement for washout of the needles used for FNAB. ${ }^{2}$

\section{Prognostic Factors of PTC}

Age: Age is one of the conventional prognostic factors and it is well-known that patients with advanced age show a dire prognosis. However, cut-off ages discriminating between high-risk and low-risk groups vary according to institutions and classification systems. In the union internationale contre le cancer (UICC) staging system, ${ }^{3}$ the cut-off age was set at 45 years and in AMES, ${ }^{4}$ it was set at 
41 years for males and 51 years for females, respectively. A recent manuscript from Austria demonstrated that an age of 45 years or older significantly affected the prognosis of PTC patients. ${ }^{5}$ Fifty years was set as a cut-off in a study from Japan in which a new classification system was proposed [Cancer Institute Hospital (CIH) Classification]. ${ }^{6}$ Age was also adopted in calculating MACIS score ${ }^{7}$ as an important prognostic factor. According to our data, a cutoff age of 55 years more appropriately reflects patient prognosis than that of 45 or 50 years and an age of 55 years or older was found to independently affect both DFS and CSS of about 6000 patients with PTC. ${ }^{8,9}$

Gender: Male gender is also a classic prognostic factor ${ }^{10-}$ ${ }^{14}$ but recent publications for DTC or only PTC showed discrepant results. ${ }^{5,15-17}$ In our most recent data, male gender was recognized as an independent prognostic factor for DFS and CSS on multivariate analysis, but the significance was lower than those of other factors, including age, tumor size, and clinical node metastasis. ${ }^{9}$ It is therefore suggested that male gender reflects patient prognosis to some extent, but its prognostic value might be weaker than those of other conventional prognostic factors.

Tumor size: Tumor size is adopted as a prognostic factor in AMES, ${ }^{4}$ MACIS, ${ }^{7}$ and UICC. ${ }^{3}$ In AMES, elderly patients having DTC larger than $5 \mathrm{~cm}$ are classified as high risk and in the T classification of UICC, cut-off sizes are set at $2 \mathrm{~cm}$ (between $\mathrm{T} 1$ and T2) and $4 \mathrm{~cm}$ (between T2 and T3). Among studies only for PTC, one showed a negative result for a prognostic value of tumor size, ${ }^{15}$ but multiple studies demonstrated its prognostic value to various degrees. ${ }^{5,16-18}$ On the basis of our analysis, PTC larger than $4 \mathrm{~cm}$ was associated with significantly worse DFS and CSS not only on univariate but also on multivariate analysis. ${ }^{8,9,19,20}$ On the other hand, PTC measuring $1 \mathrm{~cm}$ or less does not grow or grows slowly and observation without immediate surgery at diagnosis is recommended unless it has unfavorable features such as clinically apparent lymph node metastasis, high-grade malignancy on FNAB findings, located adjacent to the trachea, and located on dorsal surface possibly invading the recurrent laryngeal nerve. ${ }^{21-24}$ Another institution in Japan also recommended observation for microcarcinoma. ${ }^{25}$ Furthermore, we recently reported that hemithyroidectomy with central node dissection, instead of total thyroidectomy, is adequate for patients having solitary PTC measuring $2 \mathrm{~cm}$ or smaller without clinical lymph node or distant metastasis (T1N0M0) and radioactive iodine (RAI) therapy is not required for such patients. ${ }^{26}$ Two recent reports from Japan demonstrated that larger PTC $(>3 \mathrm{~cm}$ or $>4 \mathrm{~cm})^{27,28}$ is more likely to show recurrence to the regional nodes and prophylactic modified radical neck dissection (MND) is recommended. It is concluded that tumor size is very important to decide therapeutic strategy for PTC, including the extent of thyroidectomy and lymph node dissection and whether or not RAI therapy is performed.

Extrathyroid extension: Recent studies as well as classification systems regarded extrathyroid extension as one of the most prominent prognostic factors. ${ }^{3,4,6,7,29-32}$ It is notable that UICC TNM classification system divides extrathyroid extension into two grades; extension to sternothyroid muscle or perithyroid soft tissues (minimal extension) and extension to subcutaneous soft tissues, larynx, trachea, esophagus, recurrent laryngeal nerve, and so on (massive extension). ${ }^{3}$ Patients having carcinoma with minimal extension are upgraded to T3 and those having carcinoma with massive extension are upgraded to $\mathrm{T} 4$, the highest $\mathrm{T}$ grade, regardless of tumor size. Furthermore, UICC staging system accepts preoperative and pathological findings, but not intraoperative findings, for extrathyroid extension. Indeed, pathological evaluation of extension is often difficult and even next to impossible for various muscles, muscular coat of the esophagus, partial extension to the trachea, and recurrent laryngeal nerve requiring shaving. On the other hand, massive extrathyroid extension can be judged positive only when patients have symptoms such as hoarseness and protrusion of tumor to inner cavity of adjacent organs such as the trachea can be observed on imaging studies such as CT scan and MRI. With only such preoperative findings, minimal extension cannot be evaluated and massive extension is also quite often overlooked. Similar to other classification systems such as AMES, ${ }^{4}$ MACIS, ${ }^{7}$ and CIH classification system ${ }^{6}$ that adopt extrathyroid extension based on intraoperative findings, we also showed that intraoperative evaluation is suitable to estimate biological features of PTC, as indicated below.

In our data, intraoperative minimal extension does not reflect patient prognosis, ${ }^{33}$ whereas massive extension significantly affects DFS and CSS of patients. ${ }^{20}$ Interestingly, extrathyroid extension more strongly affects the prognosis of patients in carcinomas of larger size (our unpublished data). Conversely, in the subset of microcarcinoma, extrathyroid extension is not related to prognosis. It turns out to be one of the most prominent prognostic factors for carcinoma larger than $3 \mathrm{~cm}$.

Not only extension from primary lesions but also that from lymph node metastasis is known to predict worse 
prognosis of PTC patients. Although it is not adopted by any classification system, including UICC TNM classification, previous studies demonstrated that patients having extranodal tumor extension are more likely to show carcinoma recurrence to distant organs or die of carcinoma. ${ }^{34,35}$

Lymph node metastasis: Lymph node metastasis is the most typical biological feature of PTC and 30 to $80 \%$ of cases metastasize to the lymph nodes. There are three compartments of the regional lymph nodes of thyroid carcinoma: central, lateral, and mediastinal compartments. Lymph node metastasis is, however, not adopted as a prognostic factor in MACIS ${ }^{7}$ and AMES. ${ }^{4}$ In UICC TNM classification system, ${ }^{3}$ there are three $\mathrm{N}$ grades: N0 (no metastasis), N1a (central node metastasis), and N1b (lateral and/or mediastinal node metastasis) and prognosis is believed to get worse from N0 to N1b.

Previous studies showed discrepant data regarding the prognostic significance of lymph node metastasis. ${ }^{31,36-41}$ However, lateral lymph node metastasis detected on preoperative palpation or imaging studies such as ultrasonography (N1b) has been recognized as an independent prognostic factor for DFS and CSS of PTC patients. ${ }^{20,42}$ Actually, preoperative imaging studies frequently overlook lymph node metastasis and sensitivity of ultrasonography was low at only 22 to $29 \%$ for lateral compartment. ${ }^{9,22,41}$ For central compartment, sensitivity was even worse at $11 \% .^{9}$ However, pathologically confirmed lymph node metastasis (pN1) reflects only DFS of PTC patients and latent metastasis diagnosed only on pathological examination does not cause major problems. ${ }^{9}$ More recent study from our institution showed that, in a subset of PTC patients without massive extrathyroid extension, although N1 patients showed significantly worse DFS and CSS than N0 patients, the prognosis of N1b patients did not differ from that of N1a patients. ${ }^{43}$ However, patients having node metastasis larger than $3 \mathrm{~cm}$ and/or extranodal tumor extension requiring at least partial excision of adjacent organs (see D) are more likely to show recurrence and die of carcinoma than other N1 patients. Before the publication of our study, Sugitani et al established their own classification system to discriminate high-risk patients and node metastasis larger than $3 \mathrm{~cm}$ was one of the high-risk conditions in their system. ${ }^{6}$ In conclusion, although lymph node metastasis is a very frequent event of PTC, only clinical node metastasis of large size (e.g. $>3 \mathrm{~cm}$ ) and metastatic nodes showing extranodal extension to adjacent organs are significant prognostic factors for DFS and CSS of PTC patients. Other N1 patients might be classified as intermediate risk, that is, high incidence of recurrence but low incidence of carcinoma death.

Distant metastasis at diagnosis (M1): Although it is scarcer than that of FTC, PTC can metastasize to distant organs such as the lung, bone, and brain. Undoubtedly, M1 is the most important prognostic factor for PTC patients. ${ }^{9}$ To date, several studies have investigated the prognosis of PTC patients with distant metastasis, but most studies analyzed M1 patients and patients showing distant metastasis during follow-up after surgery as a single group or analyzed both PTC and FTC as a single DTC group. ${ }^{44-48}$ In a recent study at our institution, 71 patients with M1 PTC were analyzed and their prognoses were investigated. ${ }^{49}$ Interestingly, patients having other biological features predicting worse prognosis such as advanced age $(\geq 55$ years), large tumor size $(>4 \mathrm{~cm})$, and massive extension independently reflected CSS of M1 patients. Regarding the metastatic sites, in our data, patients showing metastasis only to lung showed a better prognosis than those with metastasis to organs other than lung. Furthermore, although no significant difference could be established, patients whose metastasis was detected on preoperative imaging studies showed a worse prognosis than those detected on postoperative whole body scan (WBS) or radioactive iodine (RAI) ablation therapy possibly because of the volume of metastasis. Patients having distant metastasis with RAI uptake, those who underwent locally curative surgery, and those who underwent TSH suppression therapy also tended to show better prognosis.

In conclusion, prognosis of M1 PTC patients can be predicted on the basis of the background of patients and clinicopathological features of primary lesions, at least to some extent.

Histological types: In the 1980s, the concept of poorly differentiated carcinoma (PDC) was proposed by Sakamoto et al, ${ }^{50}$ and Carcangiu et al. ${ }^{51}$ In 2004, PDC was adopted as an independent entity of thyroid carcinoma in the World Health Organization (WHO) classification. ${ }^{52}$ However, there are three criteria of PDC from the Japanese Society of Thyroid Surgery (JSTS), WHO classification, and Turin proposal $^{53}$ and its diagnosis is confusing. The incidence of PDC has not been studied in depth yet. To date, it is reported that the incidence of PDC based on the Turin proposal was $1.5 \%$ in North America but it was higher at 2 to $4 \%$ in Northern Italy. ${ }^{54}, 55$ In Japan, in a series of around 1700 PTC patients, the incidences of PDC based on JSTS, WHO classification, and Turin proposal were $11 \%, 0.8 \%$, and 
$0.3 \%$, respectively. ${ }^{56}$ In another study from Japan using a series of PTC and FTC patients, $15 \%, 2 \%$, and $0.3 \%$ were diagnosed as PDC on JSTS, WHO classification, and Turin proposal, respectively. ${ }^{57}$ The prognosis of PDC based on JSTS was reported to be significantly worse for DFS but not CSS on multivariate analysis. ${ }^{56}$ The prognoses of patients with PDC on WHO classification and Turin proposal were worse than PDC on JSTS but their incidences were low. Generally, PDC is not easy to diagnose preoperatively. Although there are some case reports on FNAB for PDC, no statistical evidence is available for the diagnostic accuracy for PDC on cytology.

There are various histological variants in PTC and some variants have prognostic significance. Tall cell and columnar cell variants show more adverse prognosis than conventional PTC. ${ }^{58}$ Some studies showed a worse prognosis of follicular variant ${ }^{59,60}$ but our study refused this. ${ }^{58}$ Warthin-like tumor belonging to oncocytic variant generally has a mild character, ${ }^{58}$ although one study reported a case showing anaplastic transformation. ${ }^{61}$ Diffuse sclerosing variant significantly showed clinical lymph node metastasis, but previous studies showed discrepant results for its prognosis. ${ }^{62-64}$ Our institution reported that diffuse sclerosing variant showed a worse DFS but not CSS. ${ }^{65}$ Although it is not adopted in the most recent WHO classification, encapsulated PTC was reported to show a better prognosis and the incidence of lymph node metastasis was lower than that of conventional PTC. ${ }^{66}$

\section{Therapeutic Strategies of PTC}

There are several important factors affecting patient prognosis in PTC and, fortunately, most of them can be evaluated on preoperative or intraoperative findings. Therefore, it is possible for physicians to classify each case as high-risk or low-risk before the end of surgery. In our opinion, therapeutic strategies for PTC should vary with the biological features of each case. In Western countries, routine total thyroidectomy with RAI therapy and TSH suppression has been recommended for PTC. However, as indicated above, in our experience of over 2000 patients with solitary PTC measuring $2 \mathrm{~cm}$ or less without clinical lymph node metastasis or massive extrathyroid extension, only one died of carcinoma, although about half of these patients underwent hemithyroidectomy without RAI therapy. Furthermore, high incidence of microcarcinoma without unfavorable features can be observed without immediate surgery and it is not too late to perform surgery for these patients after their tumors show progressive signs such as apparent tumor enlargement and novel appearance of node metastasis. On the basis of these findings, it appears inappropriate to perform routine total thyroidectomy with RAI therapy for such low-risk patients.

In contrast, extensive surgery followed by RAI therapy and TSH suppression is required for high-risk cases. Table 1 indicates the risk classification of TPC based on our data. All M1 patients should be classified as high-risk, although their prognoses were at least partially regulated by clinicopathological features of primary lesions. Age is the most prominent prognostic factor and studies have set various cut-off ages: 45 years in UICC classification, 41 years for males and 51 years for females in AMES, 50 years in CIH classification, and 55 years at our institution. In the subset of patients with advanced age, those having massive extrathyroid extension, large lymph node metastasis, or large tumor size have been regarded as high-risk in previous studies. Table 1 shows a summary of the risk classification of PTC according to our recent studies. All M1 patients should be classified as high-risk. Among M0 patients, those aged 55 years or older and having at least one of the following clinicopathological features are classified as high risk: (1) Tumor size larger than $2 \mathrm{~cm}$ classified as T4, (2) Lymph node metastasis larger than $3 \mathrm{~cm}$, and (3) Extranodal tumor extension requiring at least partial excision of adjacent organs. Similarly, patients aged 55 years or older and having at least one of the following features are likely to show carcinoma recurrence but not to die of carcinoma, indicating that they should be classified as intermediate risk:

(1) Tumor measuring $2 \mathrm{~cm}$ or smaller classified as T4 and

(2) Tumor size larger than $4 \mathrm{~cm}$ (excluding T4), and (3) Clinical lymph node metastasis $3 \mathrm{~cm}$ or smaller and without

Table 1: Risk classification of TPC

High-risk

a. Patients having distant metastasis at presentation

b. Patients 55 years or older and having (1), (2), or (3)

1 . Tumor size $>2 \mathrm{~cm}$ with extension to adjacent organs (corresponding to T4)

2. Lymph node metastasis $>3 \mathrm{~cm}$

3. Lymph node metastasis extending to adjacent organs Intermediate-risk

Patients 55 years or older and having (1), (2), or (3)

1. Tumor size $>4 \mathrm{~cm}$ (excluding T4 cases)

2. Tumor size $\leq 2 \mathrm{~cm}$ with extension to adjacent organs (corresponding to T4)

3. Lymph node metastasis detectable on preoperative imaging studies $\leq 3 \mathrm{~cm}$ and without extension to adjacent organs.

Low-risk

a. Patients under 55 years without distant metastasis at diagnosis

b. Patients 55 years or older who are not classified as high-risk or intermediate-risk. 
extranodal tumor extension. Extensive surgery, including total thyroidectomy and therapeutic and/or prophylactic lymph node dissection are recommended for patients with high- or intermediate-risk. For high-risk patients, in particular, adjuvant therapy such as RAI therapy and TSH suppression and careful postoperative follow-up are necessary.

\section{FTC}

The prevalence of FTC is much lower than that of PTC. In contrast to PTC, FTC is only occasionally diagnosed on preoperative cytology. Most cases are diagnosed on postoperative pathological examination based on capsular and/or vascular invasion. FTC metastasizes to distant organs rather than lymph node.

\section{Prognostic Factors of FTC}

Similar to PTC, several prognostic factors such as distant metastasis at diagnosis, tumor size, marked vascular invasion, male gender, advanced age, Hurthle cell type, lymph node metastasis, and extrathyroid extension have been identified. ${ }^{6,67-72}$ However, lymph node metastasis is rather uncommon in follicular carcinoma. One study from Germany showed that 30 of 173 patients (17\%) were classified as N1 who showed worse prognosis. ${ }^{67}$ Other studies from the United States and Austria also demonstrated that $18 \%$ and $10 \%$ of FTC patients were node-positive, respectively, but node metastasis did not independently reflect patient prognosis. ${ }^{5,68}$ In our series of 334 patients, only 11 (3\%) were positive for node metastasis. ${ }^{73}$ Since most FTC patients did not undergo lymph node dissection, the incidence would actually be higher. However, in our series, pN did not affect patient CSS and predicted a worse DFS only on univariate analysis, ${ }^{73}$ indicating that the prognostic value of lymph node metastasis for FTC is limited. Extrathyroid extension is also a rare event in FTC. In our series, only $2 \%$ of patients showed massive extrathyroid extension, corresponding to $\mathrm{T} 4 .{ }^{73}$ The incidences were $7 \%$ and $18 \%$ according to reports from the United States and Austria, respectively, ${ }^{68,5}$ but authors did not describe whether the extension was massive only or included both massive and minimal extensions. In our series, as indicated below, we failed to establish the prognostic significance of massive extrathyroid extension on multivariate analysis, which could be due to its low incidence. ${ }^{73}$ Although rare, massive extension predicts a worse prognosis of FTC patients.
Since most FTCs are diagnosed on postoperative pathological examination and conventional prognostic factors such as lymph node metastasis and extrathyroid extension are rare events, pathological examination of primary lesions is important to evaluate their biological features and prognoses.

Reports from Western countries indicate that Hurthle cell carcinoma (oxyphilic cell carcinoma) has worse prognosis, ${ }^{68,74,75}$ but discrepant results were also reported. ${ }^{70,76}$ In our series of Japanese patients, the prognoses of the $13 \%$ of them who were diagnosed with Hurthle cell type did not differ from those with conventional carcinoma. ${ }^{73}$ This result is consistent with another Japanese report. ${ }^{77}$ In 2005, a study analyzing as many as 172 patients with Hurthle cell carcinoma from the SEER database showed that histological type (Hurthle and non-Hurthle) did not reflect patient prognosis. ${ }^{78}$ Therefore, we have to conclude that the prognostic difference between Hurthle and non-Hurthle remains unclear.

In our opinion, carcinoma differentiation is very important to evaluate the prognosis of FTC patients. According to a report from Slovenia, poorly differentiated carcinoma diagnosed on the basis of Akslen's grading system had a significantly more adverse prognosis than others. ${ }^{79}$ In our series of 334 patients, 13\% of patients were diagnosed with poorly differentiated carcinoma on the basis of the WHO classification system. ${ }^{73}$ The degree of carcinoma invasion is another important factor to evaluate biological features of FTC. Cases showing grossly apparent capsular invasion (including massive extrathyroid extension) and/or marked vascular invasion are diagnosed as widely invasive FTC, while others are diagnosed as minimally invasive FTC. In our series, poorly differentiated carcinoma, widely invasive carcinoma, massive extrathyroid extension, tumor size larger than $4 \mathrm{~cm}$, distant metastasis at diagnosis, age 45 years or older and widely invasive carcinoma showed the worse CSS. Among them, poorly differentiated carcinoma and distant metastasis at diagnosis independently affected patient prognosis. Therefore, these two factors can be regarded as prominent prognostic factors of FTC patients. We graded the degree of invasiveness only when FTC was diagnosed as well-differentiated carcinoma, which is the reason for widely invasive carcinoma not being included on multivariate analysis. However, we regard widely invasive FTC as high-risk. Table 2 shows a summary of the risk classification of FTC patients. 


\begin{tabular}{|l|}
\hline \multicolumn{1}{|c|}{ Table 2: Risk classification of FTC } \\
\hline High-risk \\
- Patients diagnosed with poorly differentiated carcinoma \\
- Patients diagnosed with widely invasive carcinoma \\
- (including T4) \\
Intermediate-risk \\
- Patients 45 years or older \\
- Patients having tumor $>4$ cm \\
- Patients having lymph node metastasis \\
Low-risk \\
Patients who are not classified as high-risk or intermediate-risk. \\
\hline
\end{tabular}

\section{Therapeutic Strategy for FTC}

As indicated above, FTC is usually diagnosed on postoperative pathological examination, indicating that most patients undergo only hemithyroidectomy in initial surgery, except for those having clinical lymph node metastasis and/ or massive extrathyroid extension on preoperative or intraoperative findings. Therefore, it is debatable whether additional surgery such as completion total thyroidectomy followed by RAI therapy is necessary. In Western countries, completion total thyroidectomy is recommended when patients are diagnosed with FTC after hemithyroidectomy. However, in our series of 334 patients, 10-year CSS of patients with minimally invasive FTC and well-differentiated carcinoma were $97 \%$ and $94 \%$, respectively, while those of patients with widely invasive FTC and poorly differentiated FTC were $84 \%$ and $71 \%$, respectively. ${ }^{73}$ On the basis of these data, we conclude that additional surgery is necessary when patients are pathologically diagnosed with poorly differentiated carcinoma or widely invasive FTC. The most prominent cause of death of FTC patients is distant metastasis and RAI therapy or WBS should be prepared for such patients. However, minimally invasive FTC without poor differentiation does not require completion of total thyroidectomy as the second surgery. Unlike PTC, postoperative pathological examination is therefore important to predict patient prognosis and decide therapeutic strategies for FTC.

\section{ATC}

ATC is believed to arise from PTC and FTC and is one of the most aggressive human neoplasms. Even though patients undergo multimodal therapy such as surgery, chemotherapy, and radiotherapy, their mortality reaches at least $90 \%$.

\section{Prognostic Factors of ATC}

To date, several prognostic factors of ATC such as tumor size, patient age, distant metastasis, surgical curativity, and leukocytosis have been identified. ${ }^{80-84}$ Sugitani et al proposed a prognostic index (PI) based on the number of four unfavorable characteristics: Acute symptoms, large tumor (> $5 \mathrm{~cm}$ ), distant metastasis, and leukocytosis (white blood cell count $>10,000 / \mathrm{mm}^{3}$ ). ${ }^{83}$ They showed that patients with PI $<1$ could expect long-term survival, while no patients with PI $>3$ survived longer than 6 months.

UICC staging system for ATC consisted of three groups: Stage IVA, tumor limited to the thyroid, Stage IVB, tumor extends beyond the thyroid capsule, and Stage IVC, tumor with distant metastasis. ${ }^{3}$ Our data indicated that most Stage IVA patients underwent locally curative surgery and comparably favorable prognosis, although less than $40 \%$ of patients survived for 2 years after diagnosis. ${ }^{85}$ The prognosis of Stage IVC patients is very poor and all patients died of carcinoma within 1 year regardless of local curativity. The prognosis of Stage IVB patients depends significantly on surgical curativity. The CSS of Stage IVB patients with curative surgery did not differ from that of Stage IVA patients, while Stage IVB patients with no or only palliative surgery had as a dire prognosis as Stage IVC patients. Therefore, whether curative surgery can be performed significantly reflects the prognosis of ATC patients without distant metastasis at diagnosis. In other words, debulking surgery may temporarily reduce symptoms of patients but is considered ineffective for prolonging their survival, although a discrepant result was reported from another institution. ${ }^{86}$ In view of this finding, appropriate therapeutic strategies for ATC patients are discussed in the next section.

\section{Therapeutic Strategies for ATC}

Table 3 shows a summary of the risk classification of ATC patients. In order to prolong patient survival, multimodal therapy, surgery with radiotherapy and chemotherapy, is mandatory for ATC in any stage. As indicated above, however, long-term survival is expected for ATC patients only when carcinoma does not metastasize to distant organs and locally curative surgery is achieved. Thus, patients with Stage IVA on imaging studies whose tumors are intrathyroidal should initially undergo surgery followed by radiotherapy and/or chemotherapy. It is debatable whether surgery should initially be performed for Stage IVB patients. If curative surgery can be performed, favorable prognosis might be expected, but if not, local recurrence is expected to occur immediately. In 2000, Ain et al demonstrated that 96 hours infusion of paclitaxel is effective for ATC patients. ${ }^{87}$ Recently, weekly regimens of paclitaxel were developed to increase treatment efficacy and decrease 
Table 3: Risk classification of ATC

All patients should be classified as high-risk

Patients who can expect long-term survival.

- Stage IVA patients

- Stage IVB patients who can undergo locally curative surgery

- Stage IVB patients who can be downstaged and undergone curative surgery on induction chemotherapy

toxicity and weekly paclitaxel at $80 \mathrm{mg} / \mathrm{ml}^{2}$ is established for various carcinomas. A trial of induction chemotherapy with weekly paclitaxel has been reported and its response rate (CR+PR) was $33 \% .^{88}$ Furthermore, CSS of Stage IVB patients with weekly paclitaxel was significantly better than that of those without. Therefore, induction therapy with weekly paclitaxel followed by curative surgery after the downstaging of carcinoma can be regarded as an important strategy for Stage IVB patients. Unfortunately, no potent therapeutic strategy has been established for Stage IVC patients. The response rate for weekly paclitaxel was $25 \%$ and the clinical benefit rate (CR + PR + SD) was 75\%, but all Stage IVC patients died of carcinoma within 8 months. Other chemotherapies for multiagent chemotherapy should be investigated.

\section{CONCLUSIONS}

PTC is easy to diagnose on preoperative FNAB and the biological character of each patient can be evaluated on imaging studies and/or from intraoperative findings. As shown in Table 1, PTC having extrathyroid extension, large lymph node metastasis ( $>3 \mathrm{~cm}$ ), extranodal tumor extension, or distant metastasis at diagnosis should be regarded as highrisk and, for these patients, extensive surgery followed by RAI therapy and TSH suppression is required. In contrast, for solitary T1N0M0 patients, total thyroidectomy and RAI therapy are not mandatory and their prognoses are excellent even when they undergo hemithyroidectomy with central node dissection only. Furthermore, observation without immediate surgery can be a strong alternative for patients having PTC measuring $1 \mathrm{~cm}$ or less unless they have unfavorable features as indicated above. In Western countries, therapy for PTC tends to be uniform, but on the basis of these data, we claim that excess therapy should be avoided for low-risk cases.

In contrast to PTC, FTC is difficult to diagnose on preoperative imaging and cytological studies unless distant metastasis is present at diagnosis. The evaluation of degree of vascular and capsular invasion and carcinoma differentiation on pathological examination is quite important to predict patient prognosis and decide further therapies. We tentatively conclude that patients diagnosed with widely invasive or poorly differentiated carcinoma should undergo at least completion total thyroidectomy as a second surgery followed by RAI therapy and TSH suppression. Otherwise, second surgery is not mandatory for FTC. Patients having clinical lymph node metastasis or massive extension on preoperative and/or intraoperative findings should undergo total thyroidectomy in initial surgery.

Although various combined modality treatments have been tried, the prognosis of ATC patients is still very poor. It is no exaggeration to say that long-term survival can be expected only for patients who do not have distant metastasis at diagnosis and undergo locally curative surgery. Locally curative surgery can be performed for most Stage IVA patients, which should be the initial treatment. Radiotherapy for local control and chemotherapy as a systemic adjuvant treatment are also recommended thereafter. For most Stage IVB patients, locally curative surgery is difficult. For such patients, downstaging by induction chemotherapy or radiotherapy is recommended. In our institution, induction chemotherapy with weekly paclitaxel administration has shown relatively successful results and responders among Stage IVB patients are significantly better than nonresponders. For Stage IVC patients, regrettably, no effective modalities have yet been found.

\section{REFERENCES}

1. Antonelli A, Miccoli P, Ferdeghini M, et al. Role of neck ultrasonography in the follow-up of patients operated on for thyroid cancer. Thyroid 1995;5:25-28.

2. Uruno T, Miyauchi A, Shimizu K, et al. Usefulness of thyroglobulin measurement in fine-needle aspiration biopsy specimens for diagnosing cervical lymph node metastasis in patients with papillary thyroid cancer. World J Surg 2005;29:483-85.

3. Sobin LH, Wittekind Ch (Eds). UICC: TNM classification of malignant tumors (6th ed). New York: Wiley-Liss, 2002.

4. Cady B, Rosai R. An expanded view of risk group definition in differentiated thyroid carcinoma. Surgery 1988;104:947-53.

5. Passler C, Scheuba C, Prager G, et al. Prognostic factors of papillary and follicular thyroid cancer: Differences in an iodinereplete endemic goiter region. Endocr Relat Cancer 2004;11: 131-39.

6. Sugitani I, Kasai N, Fujimoto Y, Yanagisawa A. A novel classification system for patients with PTC: Addition of the new variable of large (3 $\mathrm{cm}$ or greater) nodal metastases and reclassification during the follow-up period. Surgery 2004;135:139-48.

7. Hay ID, Bergstrahl EJ, Goellner JR, Ebersold JR, Grant CS. Predicting outcome in papillary thyroid carcinoma: Development 
of a reliable prognostic scoring system in a cohort of 1779 patients surgically treated at one institution during 1940 through 1989. Surgery 1993;114:1050-58.

8. Ito Y, Kakudo K, Hirokawa M, et al. Biological behavior and prognosis of familial papillary thyroid carcinoma. Surgery 2009;145:100-05.

9. Ito Y, Miyauchi A. Prognostic factors and therapeutic strategies for differentiated carcinoma of the thyroid. Endocrine $\mathrm{J}$ 2009;56:177-92.

10. Mazzaferri EL, Jhiang SM. Long-term impact of initial surgical and medical therapy on papillary and follicular thyroid cancer. Am J Med 1994;97:418-28.

11. Akslen LA, Haldorsen T, Thoressen SO, et al. Survival and causes of death in thyroid cancer: A population-based study of 2479 cases for Norway. Cancer Res 1991;51:1234-41.

12. Sciuto R, Romano L, Rea S, Marandino F, Sperduti I, Maini CL. Natural history and clinical outcome of differentiated thyroid carcinoma: A retrospective analysis of 1503 patients treated at a single institution. Ann Oncol 2009;20:1728-35.

13. Shah JP, Loree TR, Dharker D, Strong EW, Begg C, Vlamis V. Prognostic factors in differentiated carcinoma of the thyroid gland. Am J Surg 1992;164:658-61.

14. Levi F, Randimbison L, Te VC, La Vecchia C. Thyroid cancer in Vaud, Switzerland: An update. Thyroid 2002;12:163-68.

15. Cheema Y, Repplinger D, Elson D, Chen H. Is tumor size the best predictor of outcome for papillary thyroid cancer? Ann Surg Oncol 2006;13:1524-28.

16. Pelizzo MR, Boschin IM, Toniato A, et al. Papillary thyroid carcinoma: 35-year outcome and prognostic factors in 1858 patients. Clin Nucl Med 2007;32:440-44.

17. Siironen P, Louhimo J, Nordling S, Ristimaki A, Maenpaa H, Haapiainen R, Haglund C. Prognostic factors in papillary thyroid cancer: An evaluation of 601 consecutive patients. Tumour Biol 2005;26:57-64.

18. Herrera MF, Lopez-Graniel CM, Saldana J, et al. Papillary thyroid carcinoma in Mexican patients: Clinical aspects and prognostic factors. World J Surg 1996;20:94-99.

19. Ito Y, Miyauchi A, Jikuzono T, et al. Risk factors contributing to a poor prognosis of papillary thyroid carcinoma; Validity of UICC/AJCC TNM classification and stage grouping. World J Surg 2007;31:838-48.

20. Ito Y, Tomoda C, Uruno T, et al. Prognostic significance of extrathyroid extension of papillary thyroid carcinoma: Massive but not minimal extension affects the relapse-free survival. World J Surg 2006;30:780-86.

21. Ito $\mathrm{Y}$, Uruno R, Nakano K, et al. An observation trial without surgical treatment in patients with papillary microcarcinoma of the thyroid. Thyroid 2003;13:381-88.

22. Ito $\mathrm{Y}$, Tomoda $\mathrm{C}$, Uruno $\mathrm{T}$, et al. Papillary microcarcinoma of the thyroid: How should it be treated? World J Surg 2004;28:1115-21.

23. Ito Y, Miyauchi A. A therapeutic strategy for incidentally detected papillary microcarcinoma of the thyroid. Nature Clin Pract Endocrinol Metab 2007;3:240-48.

24. Ito $\mathrm{Y}$, Miyauchi $\mathrm{A}$, Inoue $\mathrm{H}$, et al. An observation trial for papillary thyroid microcarcinoma in Japanese patients. World J Surg 2010;34:28-35.

25. Sugitani I, Toda K, Yamada K, Yamamoto N, Ikenaga M, Fujimoto Y. Three distinctly different kinds of papillary thyroid microcarcinoma should be recognized: Our treatment strategies and outcomes. World J Surg 2010. Jan 12. Epub ahead of print.

26. Ito Y, Masuoka H, Fukushima M, et al. Excellent prognosis of patients with solitary T1N0M0 papillary thyroid carcinoma who underwent thyroidectomy and elective lymph node dissection without radioiodine therapy. World J Surg 2009. Dec 30 Epub ahead of print.

27. Ito Y, Higashiyama T, Takamura Y, et al. Risk factors for recurrence to the lymph node in papillary thyroid carcinoma patients without preoperatively detectable lateral node metastasis: Validity of prophylactic modified radical neck dissection. World J Surg 2007;31:2085-91.

28. Sugitani I, Fujimoto Y, Yamada K, Yamamoto N. Prospective outcomes of selective lymph node dissection for papillary thyroid carcinoma based on preoperative ultrasonography. World J Surg 2008;32:2494-502.

29. Schnidler AM, van Melle G, Evequoz B, et al. Prognostic factors in papillary carcinoma of the thyroid. Cancer 1991;68:324-30.

30. Moreno-Egea A, Rodriguez-Gonzalez JM, Sola-Perez J, et al. Multivariate analysis of histopathological features as prognostic factors in patients with papillary thyroid carcinoma. Br J Surg 1995;82:1092-94.

31. Bellantone R, Lombardi CP, Boscherini M, et al. Prognostic factors in differentiated thyroid carcinoma: A multivariate analysis of 234 consecutive patients. J Surg Oncol 1998;68:23741.

32. Li JD, Chao TC, Weng HF, et al. Prognostic variables of papillary thyroid carcinoma with local invasion. Endocrine J 1999;46:9198.

33. Ito Y, Tomoda C, Uruno T, et al. Minimal extrathyroid extension does not affect the relapse-free survival of patients with papillary thyroid carcinoma measuring $4 \mathrm{~cm}$ or less over the age of 45 . Surg Today 2006;36:12-18.

34. Ito Y, Hirokawa M, Jikuzono T, et al. Extranodal tumor extension to adjacent organs predicts a worse cause-specific survival in patients with papillary thyroid carcinoma. World J Surg 2007;31:1196-203.

35. Yamashita H, Noguchi S, Murakami N, et al. Extracapsular invasion of lymph node metastasis. A good indicator of disease recurrence and poor prognosis in patients with thyroid microcarcinoma. Cancer 1999;86:842-49.

36. Mazzaferri EL, Young RL Papillary thyroid carcinoma: A 10-year follow-up report of the impact of therapy in 576 patients.

37. Salvasen H, Njolstad PR, Akslen LA, et al. Papillary thyroid carcinoma: A multivariate analysis of prognostic factors including an evaluation of the p-TNM staging system. Eur J Surg 1992; 158:583-89.

38. Scheumann GF, Gimm O, Wegener G, et al. Prognostic significance and surgical management of locoregional lymph node metastases in papillary thyroid cancer. World J Surg 1994; 18:559-67.

39. Bottger T, Kupp J, Gabbert HE, et al. Prognostically relevant factors in papillary thyroid cancer. Med Klin 1991;86:76-82.

40. Shaha AR, Shah JP, Loree TR. Risk group stratification and prognostic factors in papillary carcinoma of thyroid. Ann Surg Oncol 1996;3:534-38.

41. Stinmuller T, Klupp J, Rayes N, et al. Prognostic factors in patients with differentiated thyroid carcinoma. Eur J Surg 2000; 166:29-33. 
42. Ito Y, Tomoda C, Uruno T, et al. Ultrasonographically and anatomopathologically detectable node metastases in the lateral compartment as indicators of worse repalse-free survival in patients with papillary thyroid carcinoma. World J Surg 2005; 29:917-20.

43. Ito Y, Fukushima M, Tomoda C, et al. Prognosis of patients with papillary carcinoma having clinically apparent metastasis to the lateral compartment. Endocr J 2009;56:759-66.

44. DinneenSF, Valimaki MJ, Bergstralh EJ, Boellner JR, Gorman CA, Hay ID. Distant metastasis in papillary thyroid carcnoma: 100 cases observed at one institution during 5 decades. J Clin Endocrinol Metab 1995;80:2041-45.

45. Schlumberger M, Tubiana M, de Vathaire F, et al. Long-term results of treatment of 283 patients with lung and bone metastasis from differentiated thyroid carcinoma. J Clin Endocrinol Metab 1986;63:960-67.

46. Pacini F, Cetani F, Micolli P, et al. Outcome of 309 patients with metastatic differentiated thyroid carcinoma treated with radioiodine. World J Surg 1994;18:600-04.

47. Haq M, Harmer C. Differentiated thyroid carcinoma with distant metastases at presentation: Prognostic factors and outcome. Clin Endocrinol 2005;63:87-93.

48. Sugitani I, Fumimoto Y, Yamaoto N. Papillary thyroid carcinoma with distant metastases: Survival predictors and the importance of local control. Surgery 2008;143:35-42.

49. Ito Y, Masuoka H, Fukushima M, et al. Prognosis and prognostic factors of patients with papillary carcinoma showing distant metastasis at surgery (M1 patients) in Japan. Endocr J 2010 Apr 6 Epub ahead of print.

50. Sakamoto A, Kasai N, Sugano H. Poorly differentiated carcinoma of the thyroid. A clinicopathologic entity for a high risk group of papillary and follicular carcinomas. Cancer 1983; 53:1849-55.

51. Carcangiu ML, Zampi G, Rosai J. Poorly differentiated ("insular") thyroid carcinoma. A reinterpretation of Langhans' "Wuchende Struma". Am J Surg Pathol 1984;8:655-68.

52. Sobrinho-Simoes M, Carcangiu ML, Albores-Saavedra J, et al. Poorly differentiated carcinoma. In: DeLeillis RA, Lloyd RV, Heitz PU, et al (Eds). Pathology and genetics of tumors of endocrine organs. IARC Press, Lyon, 2004;73-76.

53. Valente M, Collini P, Nikiforov EY, et al. Poorly differentiated thyroid carcinoma: The Turin proposal for the use of uniform diagnostic criteria and an algorithmic diagnostic approach. Am J Surg Pathol 2007;31:1256-64.

54. Hiltzik D, Carlson DL, Tuttle RM, et al. Poorly differentiated thyroid carcinomas defined on the basis of mitosis and necrosis: A clinicopathologic study of 58 patients. Canceer 2006;106: 1286-95.

55. Volente M, Rapa I, Papotti M. Poorly differentiated thyroid carcinoma: Diagnostic features and controversial issues. Endocr Pathol 2008;19:150-55.

56. Ito Y, Hirokawa M, Fukushima M, et al. Prevalence and prognostic significance of poor differentiation and tall cell variant in papillary carcinoma in Japan. World J Surg 2008;32: 1535-43.

57. Sugitani I, Toda K, Yamamoto N, Sakamoto A, Fujimoto Y. Re-evaluation of histopathological factors affecting prognosis of differentiated thyroid carcinoma in an iodine-sufficient country. World J Surg 2009 Dec 1 Epub ahead of print.
58. Ito Y, Hirokawa M, Uruno T, et al. Prevalence and biological behaviour of variants of papillary thyroid carcinoma: Experience at a single institute. Pathology 2008;40:617-22.

59. Chag HY, Lin JD, Chou SC, et al. Clinical presentations and outcomes of surgical treatment of follicular variant of the papillary thyroid carcinomas. Jpn J Clin Oncol 2006;36: 688-93.

60. Liu J, Singh B, Tallini G, et al. Follicular variant of papillary thyroid carcinoma: A clinicopathologic study of a problematic entity. Cancer 2006;107:1255-64.

61. Lam KY, Lo CY, Wei WI. Warthin tumor-like variant of papillary thyroid carcinoma: A case with dedifferentiation (anaplastic change) and aggressive biological behavior. Endocr Pathol 2005;16:83-89.

62. Egea MA, Gonzalez RJM, Perez SF, et al. Clinicopathological study of the diffuse sclerosing variety of papillary cancer of the thyroid. Resentation of 4 new cases and review of literature. Eur J Surg Oncol 1994;20:7-11.

63. Soares J, Limbert E, Sobrinho-Simoes M. Diffuse sclerosing variant of papillary thyroid carcinoma. A clinicopathologic study of 10 cases. Pathol Res Pract 1989;185:200-06.

64. Schoroder S. Diffuse sclerosing variant of papillary thyroid carcinoma. Am J Surg Pathol 1991;15:492-93.

65. Fukushima M, Ito Y, Hirokawa M, et al. Clinicopathologic characteristics and prognosis of diffuse sclerosing variant of papillary thyroid carcinoma in Japan: An 18-year experience at a single institution. World J Surg 2009;33:958-62.

66. Ito Y, Hirokawa M, Uruno T, et al. Biological behavior and prognosis of encapsulated papillary carcinoma of he thyroid; experience of a Japanese hospital for thyroid care. World J Surg 2008;32:1789-94.

67. Witte J, Goretzki P, Dieken J, Simon D, Roher H. Importance of lymph node metastasis in follicular thyroid cancer. World J Surg 2002;26:1017-22.

68. Shaha AR, Loree TR, Shah JP. Prognostic factors and risk group analysis in follicular carcinoma of the thyroid. Surgery 1995; 118:1131-38.

69. Brennan MD, Bergstralh EJ, van Heerden JA, et al. Follicular thyroid cancer treated at the Mayo Clinic, 1946 through 1970: Initial manifestations, pathologic findings, therapy, and outcome. Mayo Clin Proc 1991;66:11-22.

70. Besic N, Zgajnar J, Hocevar M, et al. Is patient's age a prognostic factor for follicular thyroid carcinoma in the TNM classification system? Thyroid 2005;15:439-48.

71. Chow SM, Law SCK, Mendenhall WM, et al. Follicular thyroid carcinoma. Cancer 2002;95:488-98.

72. Lang W, Choritz H, Hundeshagen H. Risk factors in follicular thyroid carcinoma. Am J Surg Pathol 1986;10:246-55.

73. Ito $Y$, Hirokawa M, Higashiyama $T$, et al. Prognosis and prognostic factors of follicular carcinoma in Japan: Importance of postoperative pathological examination. World J Surg 2007; 31:1417-24

74. Yutan E, Clark OH. Hurthle cell carcinoma. Curr Treat Options Oncol 2001;2:331-35.

75. Kushchayeva Y, Duh QY, Kebebew E, et al. Comparison of clinical characteristics at diagnosis and during follow-up in 118 patients with Hurthle cell or follicular thyroid cancer. Am J Surg 2008;195:457-62.

76. Grabe SK, Hay ID. Follicular thyroid cancer. Endocrinol Metab Clin North Am 1995;24:761-801. 
77. Sugino K, Ito K, Mimura T, et al. Hurthle cell tumor of the thyroid: Analysis of 188 cases. World J Surg 2001;25:1160-63.

78. Haigh PI, Urbach DR. The treatment and prognosis of Hurthle cell follicular thyroid carcinoima compared with its non-Hurthel cell counterpart. Surgery 2005;138:1152-57.

79. Besic N, Auersperg M, Golouh R. Prognostic factors in follicular carcinoma of the thyroid: A multivariate survival analysis. Eur J Surg Oncol 1999;25:599-605.

80. Venkatesh YS, Ordonez NG, Schultz PN, et al. Anaplastic carcinoma of the thyroid: A clinicopathologic study of 121 cases. Cancer 1990;66:321-30.

81. Kebebew E, Greenspan FS, Clark OH, et al. Anaplastic thyroid carcinoma. Treatment outcome and prognostic factors. Cancer 2005;103:1330-35.

82. Lo CY, Lam KY, Wan KY. Anaplastic carcinoma of the thyroid. Am J Surg 1999;177:337-39.
83. Sugitani I, Kasai N, Fujimoto Y, et al. Prognostic factors and therapeutic strategy for anaplastic carcinoma of the thyroid. World J Surg 2001;25:617-22.

84. Kihara M, Miyauchi A, Yamauchi A, et al. Prognostic factors in anaplastic carcinoma. Surg Today 2004;34:394-98.

85. Ito $\mathrm{Y}$, Higashiyama $\mathrm{T}$, Hirokawa $\mathrm{M}$, et al. Investigation of the validity of UICC stage grouping of anaplastic carcinioma of the thyroid. Asian J Surg 2009;32:47-50.

86. Sugino K, Ito K, Mimura T, et al. The important role of operations in the management of anaplastic thyroid carcinoma. Surgery 2002;131:245-48.

87. Ain KB, Egorin MJ, DeSimone PA. Treatment of anaplastic thyroid carcinoma with paclitaxel: Phase 2 trial using ninetysix-hour infusion. Tyroid 2000;10:587-94.

88. Higashiyama $\mathrm{T}$, Ito $\mathrm{Y}$, Hirokawa $\mathrm{M}$, et al. Induction chemotherapy with weekly paclitaxel administration for anaplastic thyroid carcinoma. Thyroid 2010;20:7-14. 